\title{
Molecular Structures of Yeast Kinetochore Subcomplexes and Their Roles in Chromosome Segregation
}

\author{
Simon Jenni, ${ }^{1}$ Yoana N. Dimitrova, ${ }^{1,2}$ Roberto Valverde, ${ }^{1,3}$ Stephen M. Hinshaw, ${ }^{1,2}$ \\ and Stephen C. HaRrison ${ }^{1,2}$ \\ ${ }^{1}$ Department of Biological Chemistry and Molecular Pharmacology, Harvard Medical School, \\ Boston, Massachusetts 02115 \\ ${ }^{2}$ Howard Hughes Medical Institute, Boston, Massachusetts 02115 \\ Correspondence: harrison@crystal.harvard.edu
}

\begin{abstract}
Kinetochore molecular architecture exemplifies "form follows function." The simplifications that generated the one-chromosome:one-microtubule linkage in point-centromere yeast have enabled strategies for systematic structural analysis and highresolution visualization of many kinetochore components, leading to specific proposals for molecular mechanisms. We describe here some structural features that allow a kinetochore to remain attached to the end of a depolymerizing microtubule (MT) and some characteristics of the connections between substructures that permit very sensitive regulation by differential kinase activities. We emphasize in particular the importance of flexible connections between rod-like structural members and the integration of these members into a compliant cage-like assembly anchored on the MT by a sliding molecular ring.
\end{abstract}

Kinetochores attach chromosomes to microtubules (MTs) of the mitotic spindle, thereby coupling chromosome movement and MT dynamics. Studies of the molecular organization of kinetochores became possible when Carbon and coworkers showed that budding-yeast centromeres are relatively short, $\sim 125$-bp, regions with defined DNA sequences (Clarke and Carbon 1980) recognized by specific proteins, some of which they proceeded to isolate (Lechner and Carbon 1991). These proteins in turn direct deposition of a single, centromere-specific nucleosome (Meluh et al. 1998). The "parts list" for the kinetochores that then assemble on the so-called "point centromeres" of budding yeast now includes more than 50 distinct gene products (depending on how one chooses to define a "part"), most of them associated into distinct, multiprotein complexes (De Wulf et al. 2003; Musacchio and Desai 2017). Conservation of many of these complexes among eukaryotes has reinforced the view that a budding-yeast kinetochore represents a simplified module of the kinetochores distributed over much longer centromeres in other organisms (Fig. 1).

\section{MT ATTACHMENT}

Kinetochore protein complexes fall into three groups: MT-proximal components, chromatin-proximal components, and intermediate adaptors. We concentrate in this report on some molecular-structural characteristics of MT attachment and adaptor interactions. Two of us have reviewed elsewhere the molecular activities of chromatinproximal components (Hinshaw and Harrison 2017). Because point-centromere kinetochores capture a single spindle MT (Winey et al. 1995), they create a one-to-one centromere-to-MT bridge. The molecular architecture of the kinetochore-MT connection is thus more easily described than it is for kinetochores on the larger, multinucleosomal, "regional" centromeres of most other eukaryotes. Ndc80c and DASH/Dam $1 \mathrm{c}$ are the principal MT attachment complexes (Fig. 1). Ndc80c is a long $(\sim 620-\AA)$ rod with small, globular ends (Ciferri et al. 2005; Wei et al. 2005). The shaft of the rod is largely a parallel, two-chain $\alpha$-helical coiled-coil. DASH/Dam1c encircles the MT by assembling into a sliding ring (Miranda et al. 2005; Westermann et al. 2005). It is likely that the ring is both a processivity factor and an organizer for the approximately eight $\mathrm{Ndc} 80 \mathrm{c}$ rods (Joglekar et al. 2006) that extend from each kinetochore to capture the plus end of a single MT.

\section{Ndc80c}

The complex is a heterotetramer (Fig. 2A; Ciferri et al. 2005; Wei et al. 2005). Ndc80 and Nuf2 pair at one end and contribute $\sim 70 \%$ of the coiled-coil shaft. An extended segment of about 100 amino acid residues at the amino terminus of Ndc80 and the calponin homology $(\mathrm{CH})$ domain that follows it together create the MT contact (Cheeseman et al. 2006; Wei et al. 2007); a CH domain at the amino terminus of Nuf2 supports the MT contacting module but does not reach the MT surface (Ciferri et al. 2008). Spc24 and Spc25 pair at the other end of the rod, with the same N-to-C polarity as Ndc80:Nuf2, so that the globular tip contains the tightly associated, carboxy-terminal, RWD domains of the two subunits (Wei et al. 2006).

\footnotetext{
${ }^{3}$ Present address: Relay Therapeutics, Cambridge, Massachusetts 02142

(C) 2017 Jenni et al. This article is distributed under the terms of the Creative Commons Attribution-NonCommercial License, which permits reuse and redistribution, except for commercial purposes, provided that the original author and source are credited.
} 


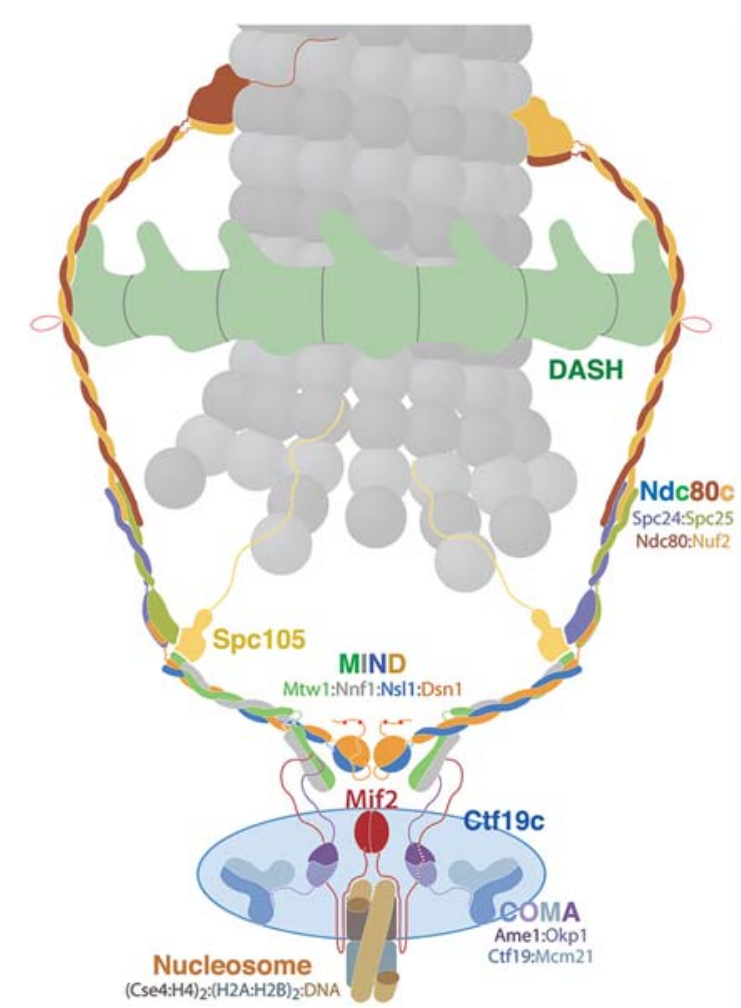

Figure 1. Schematic diagram of the principal molecular components of a yeast kinetochore. This two-dimensional representation does not show relative stoichiometries: Although there is just one centromeric (Cse4-containing) nucleosome and one microtubule, there are approximately four to six MIND complexes and approximately eight $\mathrm{Ndc} 80$ complexes, distributed, like the two shown here, around the DASH/Dam1c ring (Joglekar et al. 2006); some of the latter may be recruited by Cnn1, part of the Ctf19 complex, which includes a total of 13 components (Pekgöz Altunkaya et al. 2016). The contacts from Mif2 and Ame1 may be to distinct MIND complexes (accounting for up to four copies of MIND), rather than to the same one, as drawn for simplicity here.

The crystal structure of a "dwarf" Ndc80c, with a large deletion in the coiled-coil shaft of Ndc80:Nuf2 and a somewhat shorter deletion in the shaft of Spc24:Spc25, shows the molecular details of the end-to-end joint between the two coiled-coils (Fig. 2B; Valverde et al. 2016). The transition between a two-chain coiled-coil of Ndc80 and Nuf2 to a four-helix bundle and back to a twochain coiled-coil of Spc24 and Scp25 includes some irregularities for which the contributing amino acid residues are conserved among point-centromere fungi (Fig. 2B, inset). These idiosyncratic features suggest interaction with another, yet-to-be-determined kinetochore component.

The heptad registers established on both sides of the joint by the dwarf Ndc80c structure have allowed us to predict the structures of the deleted coiled-coiled regions. Direct coupling analysis (DCA), based on 1200 fungal $\mathrm{Ndc} 80 \mathrm{c}$ sequences, yields correlations consistent with heptad counting (Morcos et al. 2011; Ekeberg et al. 2013, 2014). Opposite Nuf2 residue 309 is an insertion in Ndc80, called the "Ndc80 loop" (Fig. 2C; Maiolica et al. 2007). Deletion of loop residues impairs end-on MT attachment but does not affect MT side binding, at least in part because of likely interactions with DASH/Dam1c (Maure et al. 2011).

Images of negatively stained Ndc80c suggest a tendency to bend at about $160 \AA$ from the Ndc80:Nuf2 globular tip (Wang et al. 2008). In previous work, this "kink" has been assigned to the loop position, but the analysis here suggests that the mean position of the loop should be at $\sim 270 \AA$ from the tip instead (Fig. 2C). Preferential proteolytic cleavages at residues 380 and 410 in Ndc80 indicate some variation in the strength of coiled-coil interactions along the Ndc80:Nuf2 shaft; the positions of cleavage would be at about $160 \AA$ and $200 \AA$ from the tip, respectively (Wei et al. 2005). Measurements performed on recently published images of rotary-shadowed Ndc80c are more consistent with a somewhat distributed tendency to bend, rather than a uniquely positioned kink (Huis In 't Veld et al. 2016). An additional point of local flexibility is at the head-shaft junction, as indicated by comparison of crystal structures of two different dwarf Ndc 80 c constructs (Valverde et al. 2016). These properties may allow Ndc80c to bend around the DASH/Dam1c ring, as suggested schematically in Figure 1.

The MT interface of the Ndc 80 head includes both its $\mathrm{CH}$ domain and amino-terminal extension; the $K_{\mathrm{d}}$ for the human ortholog (Hec1) is $\sim 2 \mu \mathrm{M}$. Phosphorylation by Aurora B/Ipl1 or deletion of the extension weakens the interaction, raising the $K_{\mathrm{d}}$ by about an order of magnitude (DeLuca et al. 2006; Wei et al. 2007). Bead and singlemolecule tracking experiments have shown that $\mathrm{Ndc} 80 \mathrm{c}$ in clusters of two or more diffuses on the surface of a MT lattice (Powers et al. 2009). A biased diffusion mechanism can then account for in vitro measurements of assemblyand disassembly-coupled movement, perhaps reflecting the properties of initial, side-on MT capture by kinetochores, which depends on Ndc80c but not on DASH/ Dam1c (Tanaka et al. 2005, 2007).

\section{DASH/Dam1c}

The DASH/Dam1c protomer is an assembly of 10 distinct polypeptide chains, all of which are needed to reconstitute the complex in vitro (Li et al. 2002; Miranda et al. 2005). In the presence of MTs, it encircles them by polymerizing into rings of about twice the outer diameter of the MT (Miranda et al. 2005; Westermann et al. 2005). The MT contacts are from extended carboxy-terminal "arms" of two of the subunits, Dam1 and Duo1 (Miranda et al. 2007). Redundancy and flexibility of these contacts can allow the ring to track the end of the MT, by rapidly detaching and reattaching.

Moderate-resolution cryo-EM reconstructions of DASH/Dam1c rings show 15- and 16-protomer assemblies when reconstituted free in solution (at relatively high protein concentration) or around MTs, respectively (Ramey et al. 2011). The protomer has the shape of a thick rod, with a short protrusion at its center. Analysis of the amino acid sequences of the ten subunits and of their conservation among fungal species suggests that the body of the protomer contains largely $\alpha$-helical regions of the com- 
A

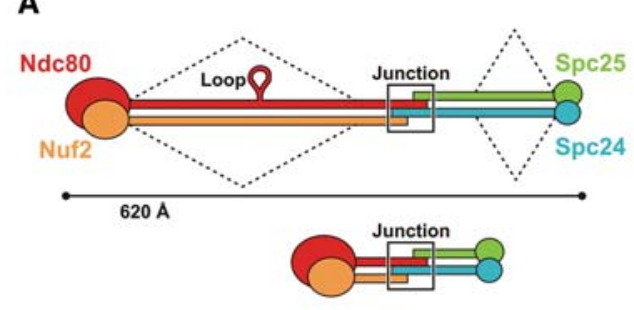

B

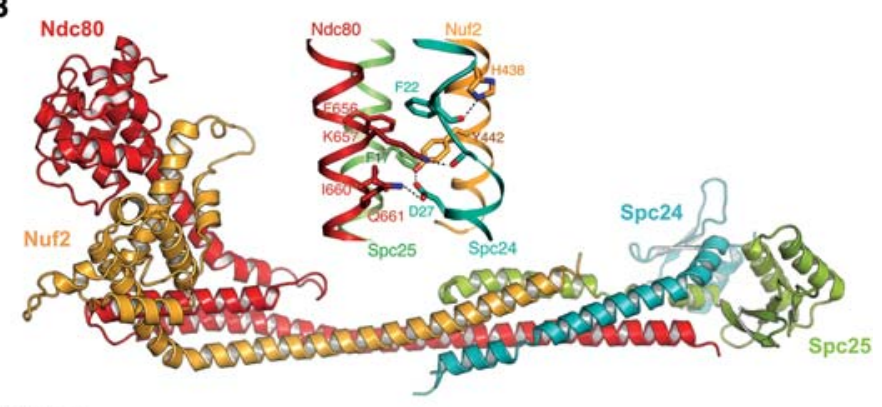

C

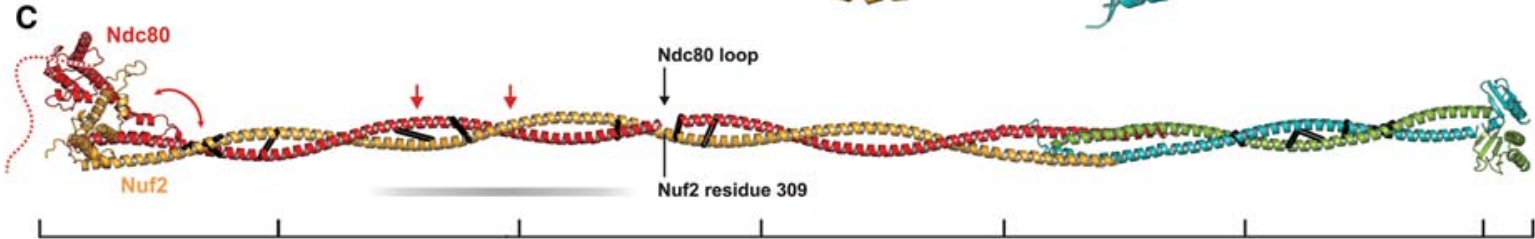

Figure 2. Structure of Ndc80c. (A) Diagram of Ndc80c subunit organization. Dashed lines show deletions used to create a dwarf Ndc80c that retains the tetramer junction. (B) Structure of dwarf Ndc80c (Saccharomyces cerevisiae) (Valverde et al. 2016). Inset shows detail of the junction region, with residues conserved in point-centromere yeast shown as sticks on the ribbon backbone. $(C)$ Model of complete Ndc80c structure, based on the structure of dwarf Ndc80c, heptad counting across the deleted coiled-coil segments, and inferences from published cross-linking data for the human ortholog (Maiolica et al. 2007). Results of direct-coupling analysis (see text) are shown as heavy black lines. The scale bar below corresponds to $620 \AA$, with $100-\AA \AA$ intervals marked. Dotted red curve, amino-terminal extension of Ndc80; red arrowheads, preferential cleavage points in Ndc80 (Wei et al. 2005); curved, double-headed arrow, region of hinge-like joint indicated by comparison of two different dwarf constructs; and gray bar, approximate distribution of bends in published rotaryshadowed images (Huis In't Veld et al. 2016: Fig. 6, figure supplement 2) of human Ndc80c (140 particles; 72 with visually identifiable kink or bend; 38 unbent; 30 poorly contrasted or not measurable; mean position of bend $\sim 200 \AA$ from one end). ( $A, B$, Modified, with permission, from Valverde et al. 2016.)

ponents, with long, potentially flexible extensions at the carboxy-terminal ends of Dam1, Duo1, and Ask1, consistent with their protease sensitivity, and shorter extensions at the amino termini of many of the subunits (Fig. 3).

Chemical cross-linking of Ndc80 with DASH/Dam1c, analyzed by mass spectrometry, has defined three sets of cross-linked contact points (Kim et al. 2017). Residues near the carboxyl terminus of Dam1 cross-link with residues in the helical hairpin connecting the $\mathrm{CH}$ domain of
Nc80 with the coiled-coil shaft; residues in the carboxyterminal half of Ask1 cross-link with residues in $\mathrm{Ndc80}$ between the helical hairpin and the loop; and residues in the carboxy-terminal half of Spc34 cross-link with residues in Ndc80 near the junction with Spc24:Spc25. These positions on Ndc80 span $\sim 300 \AA$ along the coiled-coil shaft. In vivo fluorescence resonance energy transfer (FRET) analysis also places the carboxyl terminus of Dam1 close to the globular end of Ndc80:Nuf2 (Arava-

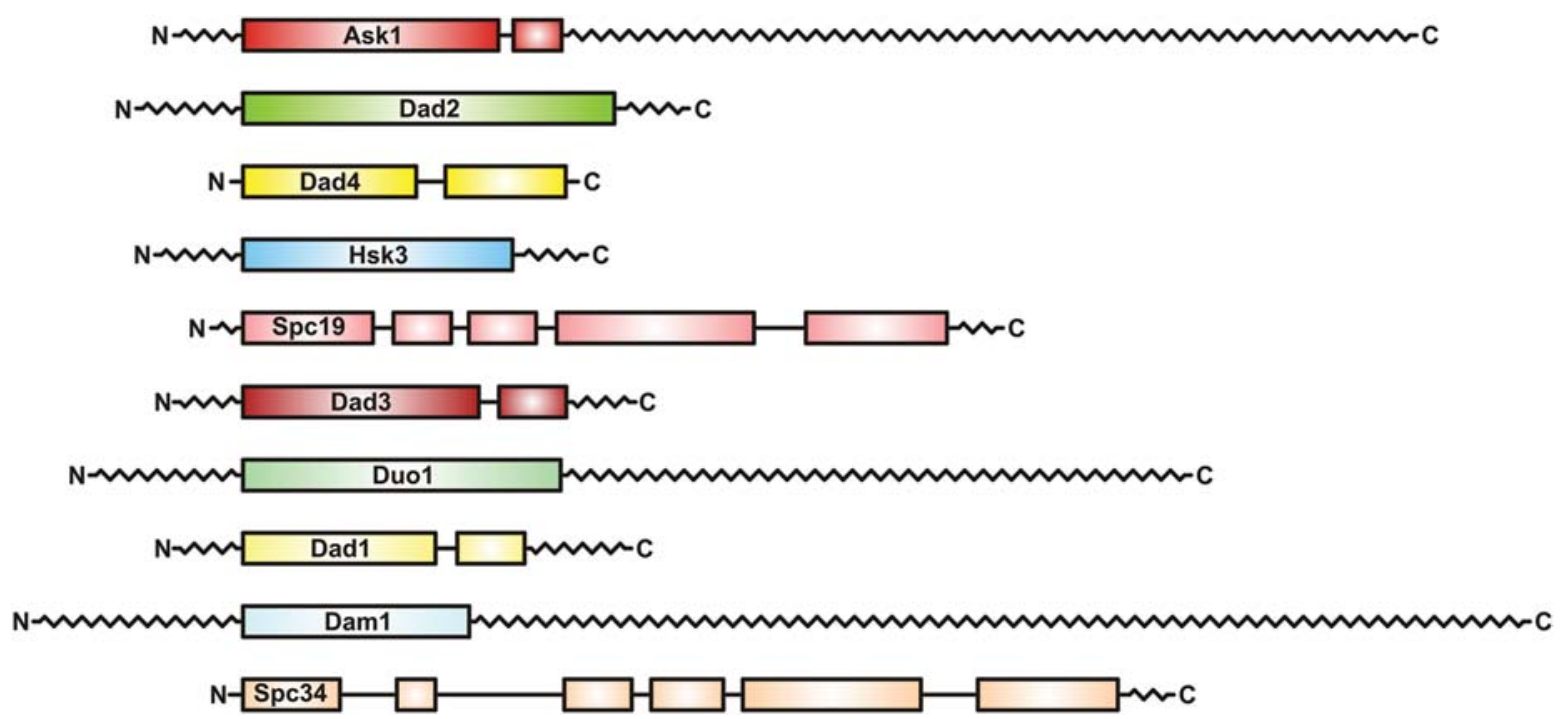

Figure 3. DASH/Dam1c subunits. Boxes show regions of predicted fold, largely $\alpha$-helical; zig-zags show prediction of flexible aminoand carboxy-terminal extensions. 
mudhan et al. 2014). Moreover, the extensions of Dam1, Duo1, and Ask1 are long enough (Fig. 3) that flexible projections from even a single, relatively compact ring could span the required distance, so that all the cross-links could originate from concurrent contacts by the same ring and even by the same protomers in that ring. Indeed, the reported amounts per cell of several of the $10 \mathrm{DASH} / \mathrm{Dam} 1 \mathrm{c}$ components are enough for just one 15- or 16-protomer ring per kinetochore MT (Ghaemmaghami et al. 2003).

A stable transition from side-on to end-on MT attachment requires DASH/Dam1c (Tanaka et al. 2007). The geometry of end-on attachment couples the curled ends of disassembling protofilaments with the position of the attached kinetochore (Asbury et al. 2006), and MT depolymerization, rather than motor activity, can indeed drive poleward motion of chromosomes in anaphase (Koshland et al. 1988; Grishchuk and McIntosh 2006; Tytell and Sorger 2006). The diameter of the DASH ring will prevent it from pulling off the MT, and recent laser-trap experiments have shown that the curled protofilaments have enough elastic strength to exert the required force (Driver et al. 2017). Flexible connections from the carboxy-terminal extensions of Dam1 and Duo1 will facilitate biased motion in response to MT depolymerization, as protofilament curling will experience relatively little resistance. Moreover, because an individual arm can reach multiple tubulin binding sites, dissociation and reassociation of just one or a few of these contacts will allow the ring (and the attached chromosome) to translocate (Miranda et al. 2007).
DASH/Dam1c is present primarily in fungi, but also in representative species of other eukaryotic taxa (van Hooff et al. 2017). Most of the species, including nearly all metazoans, that lack DASH/Dam1c have the three-subunit Ska complex instead, and very few show evidence of both. Tracking the two complexes back along the evolutionary tree appears to favor a model in which Ska was present in the last eukaryotic common ancestor and that DASH/ Dam1c appeared in an ancestral fungal species, displacing Ska and spreading to certain other eukaryotes by lateral transfer (van Hooff et al. 2017). The authors of the evolutionary analysis have suggested that the two structurally unrelated complexes are functional analogs and that both coordinate Ndc80c attachment to spindle MTs.

\section{ADAPTORS}

Two adaptor complexes-MIND ${ }^{\mathrm{MIS} 12 \mathrm{c}}$ and Cnn1: Wip1 CENP-T:CENP-W_ _ associate with the Spc24:Spc25 tip of Ndc80c and connect the MT attachment assembly with chromatin-associated complexes (Hori et al. 2008; Malvezzi et al. 2013). The former is essential in yeast; the latter is not. Thus, Cnn1:Wip1, presumably with associated Ndc80 complexes, may enhance kinetochore strength or connectivity but probably does not modify its fundamental molecular organization. The two adaptors bind Spc24:Spc25 through related motifs - at the carboxy-terminal end of the MIND subunit Dsn1 and near the aminoterminal end of Cnn1 (Bock et al. 2012; Malvezzi et al. 2013; Dimitrova et al. 2016; Petrovic et al. 2016). Mps1

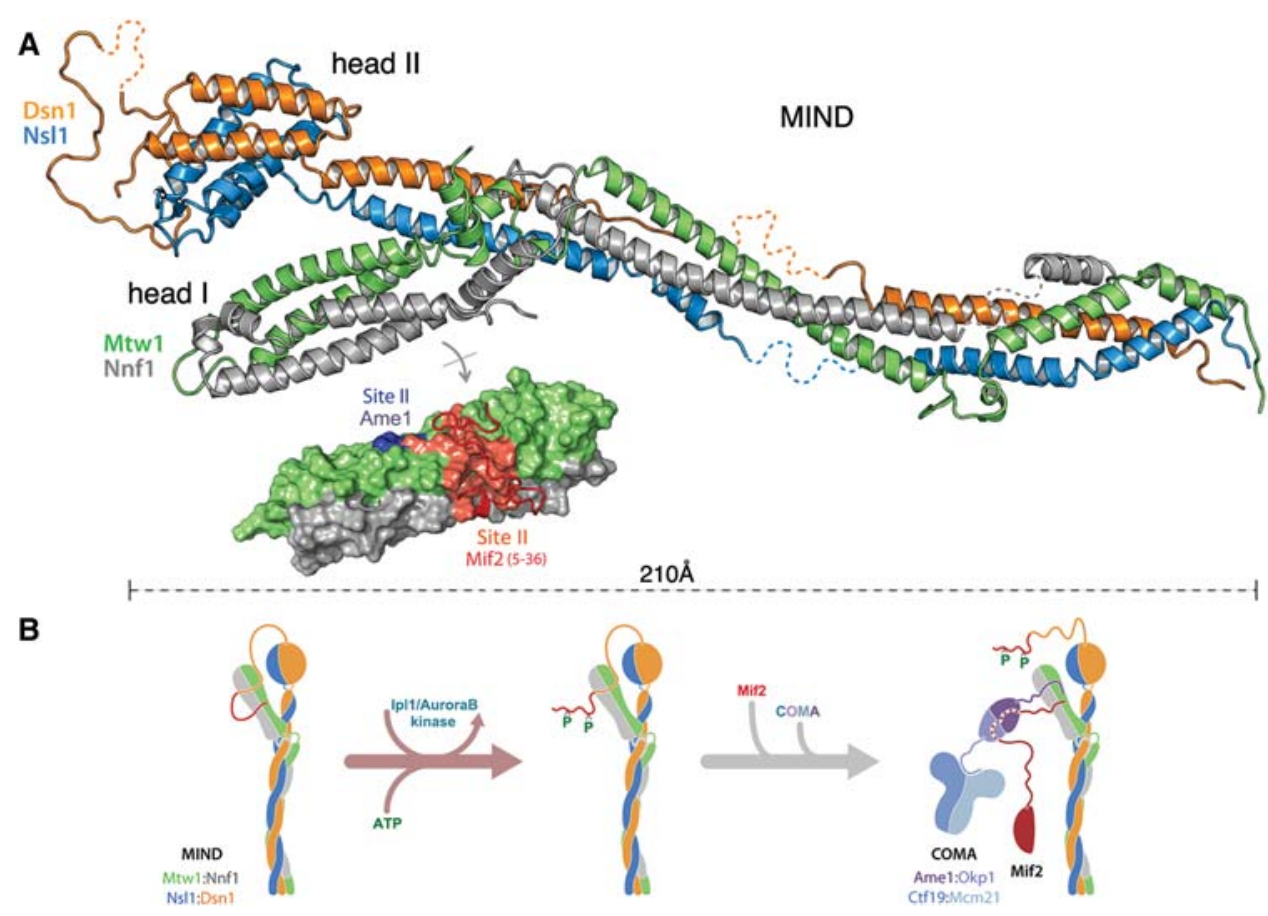

Figure 4. Structure and regulation of MIND. $(A)$ Ribbon diagram of the MIND heterotetramer with surface representation of head I. Positions of Mif2 and Ame1 binding are shown as patches of red and blue, respectively; the backbone of Mif2 (residues 5-36) is shown in its bound conformation. $(B)$ Scheme showing mechanism by which phosphorylation of Dsn1 relieves MIND autoinhibition, allowing Mif2 and Ame1 to interact with head I. (Modified, with permission, from Dimitrova et al. 2016; original structure generated with PyMOL [http://www.pymol.org].) 
phosphorylation of a serine residue in the binding segment of Cnn1 blocks the interaction (Thapa et al. 2015); serine residues in the related Dsn 1 segment are potential sites of phosphoregulation, but a role for their modification, if any, has yet to be identified (Dimitrova et al. 2016).

MIND is a $210-\AA$-long, heterotetrameric rod, with two globular, four-helix bundles ("heads") at one end (Dimitrova et al. 2016; Petrovic et al. 2016). Mtw1 ${ }^{\text {MIS12 }}$ and $\mathrm{Nnfl}^{\mathrm{PMF1}}$ contribute to "head 1"; Dsn1 and Ns11, to "head 2." Head 1 binds projecting segments from two different, chromosome-proximal kinetochore components-Mif2 ${ }^{\text {CENP-C }}$ and Ame ${ }^{\text {CENP-U }}$ (Fig. 4). Both these interactions are sensitive to phosphorylation of residues in a Dsn1 aminoterminal extension that projects from head 2 (Akiyoshi et al. 2013). When not phosphorylated, this segment of Dsn 1 binds head 1 and blocks the sites for Mif2 and Ame1 (Dimitrova et al. 2016; Petrovic et al. 2016). Phosphorylation at two positions (serines 240 and 250 in Saccharomyces cerevisiae) by $\mathrm{Ip} 11^{\text {AuroraB }}$ reverses the interaction and allows Mif2 and Ame1 to bind instead (Fig. 4B).

Ipl1 also phosphorylates the amino-terminal regions of Ndc80 and Dam1, presumably to promote their dissociation from the MT surface when sister kinetochores are not under tension from bi-orientation (Biggins et al. 1999; Cheeseman et al. 2002; Akiyoshi et al. 2009). At the level of molecular mechanism, this activity resembles the action of Ipl1 on Dsn 1 by enhancing dissociation of the modified segment from a target site. At the level of the larger assembly, the outcome is different: Ipl1 promotes MIND attachment to chromosome-proximal components by reversing an autoinhibition, but it promotes Ndc80c and DASH/ Dam1c detachment from MTs in the absence of tension (Tanaka et al. 2002; Kalantzaki et al. 2015). The specific properties of the Ndc80c-DASH/Dam1c assembly that allow it to respond to tension and protect the amino-terminal segments of Ndc80 and Dam1 from phosphorylation, either by increasing the lifetimes of their bound states or by shielding them from Ipl1, remain to be determined. The same or related structural properties might also account for the observation that tension stabilizes attachment independently of Ipl1 (Akiyoshi et al. 2010).

\section{INTERCOMPLEX CONNECTIONS}

The various complexes just described all have a conformationally well-defined core with flexibly projecting extensions, often from one or both termini of a subunit. The extensions link the complexes into larger substructures by docking into specific binding sites. These "docked-peptide" interactions (see Table 1) have functionally important properties: (1) adaptability of the contact to alternative conformational contexts, (2) mechanical compliance of the larger assembly, (3) ease of regulation by phosphorylation or other modification, and (4) potential for intervention by a disassembly-inducing modification.

1. Adaptability is the most general of these properties. In an assembly composed of multiple copies of a structure unit, but without strict symmetry, the relative orientations of connected components are necessarily variable.
Table 1. Docked-peptide interactions between yeast kinetochore subcomplexes discussed in this article

\begin{tabular}{|c|c|}
\hline Donor (docked peptide) & Acceptor (docking site) \\
\hline $\operatorname{Mif2}\left(\mathrm{N}-\right.$ term $\left.^{\mathrm{a}}\right)$ & MIND (head 1) \\
\hline COMA: Ame1 & MIND (head 1) \\
\hline MIND: Dsn1 (N-term $\left.{ }^{\mathrm{a}}\right)$ & MIND (head 1) \\
\hline MIND: Dsn1 (C-term) & Ndc80c (Spc24:Spc25) \\
\hline Cnn1 $\left(\mathrm{N}-\right.$ term $\left.^{\mathrm{a}}\right)$ & Ndc80c (Spc24:Spc25) \\
\hline DASH: Dam1 (C-term $\left.{ }^{a}\right)$ & MT \\
\hline DASH: Duo1 (C-term) & MT \\
\hline Ndc80c: Ndc80 (N-term $\left.{ }^{a}\right)$ & MT \\
\hline
\end{tabular}

${ }^{\mathrm{a}} \mathrm{Shown}$ to be regulated by phosphorylation of peptide.

N-term, amino-terminal; C-term, carboxy-terminal; MT, microtubule.

Even the stoichiometry of some of the kinetochore components may vary. Cnn1 recruitment seems to vary with cell cycle timing (Bock et al. 2012), and therefore the number of $\mathrm{Ndc} 80$ complexes probably does also. Moreover, redundancy of contacts implies that not all opportunities for recruitment need be fulfilled.

2. Mechanical compliance could in principle account for some of the kinetochore's apparent tension-sensing properties. Tension can be detected by the extent to which it displaces one part of an assembly with respect to another. The yeast kinetochore appears to be an open, cage-like structure (Akiyoshi et al. 2010); flexible connections between its rod-like members (especially Ndc80c and MIND) imply that the cage can distort (e.g., elongate or contract) without major changes within the individual subcomplexes (Fig. 5). It remains to be determined whether this property is indeed part of kinetochore-signaling mechanisms.
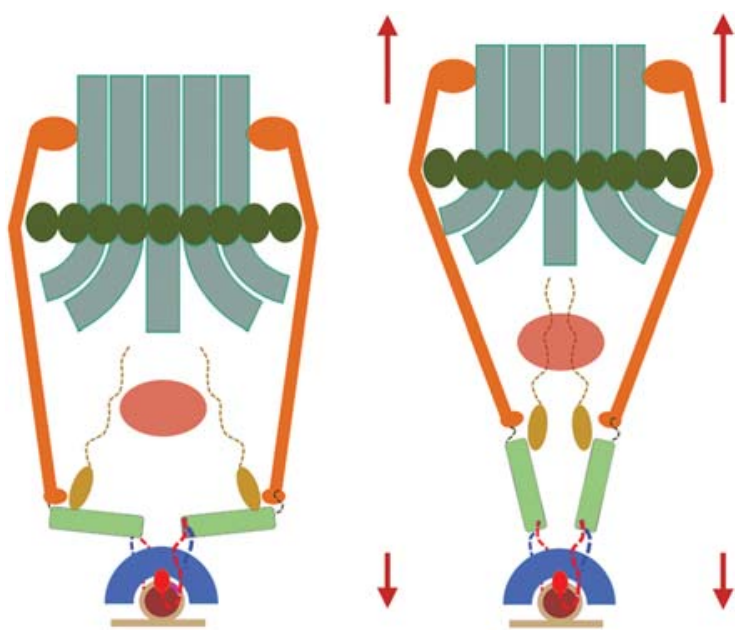

Figure 5. Example of how a kinetochore might respond to tension. (Left) Relaxed structure in the absence of tension. (Right) Extended structure in the presence of tension. Key hinge points are at docked-peptide interfaces between subcomplexes and (perhaps) at positions of preferential bending within Ndc80c (see Fig. 2). Colors of components correspond approximately to those in Figure 1; the red oval in the center symbolizes that tension can in principle affect the spindle-assembly checkpoint proteins, which associate (directly or indirectly) with Mps1-phosphorylated repeats on Spc105 (Aravamudhan et al. 2016). 


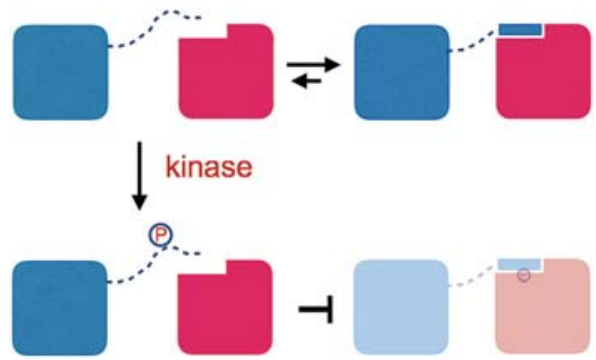

Figure 6. Docked-peptide association and reversal by phosphorylation. A flexible peptide (e.g., at the amino- or carboxyl terminus) extended from a stably folded core docks into a target site on another protein (top). When transiently dissociated, capture by a kinase phosphorylates a target residue, blocking reassociation (bottom).

3. Regulation by modification requires a segment of unfolded and extended polypeptide chain, because of the active site properties of the modifying enzymes. Protein kinases specify, by the characteristics of their substrate docking site, the sequence context of the residue to be phosphorylated (e.g., Moore et al. 2003). Switching of docked-peptide contacts by phosphorylation is therefore a familiar mode of regulation, either within a multidomain protein (e.g., the carboxy-terminal tail of c-Src) or between members of a multicomponent assembly (e.g., the amino-terminal region of Dsn1).

4. A disassembly inducing modification requires a transient dissociation ("breathing"), so that the modifier can access a residue that participates in the contact. The buried surface in a docked peptide contact is relatively modest, usually resulting in equilibrium dissociation constants of between 1 and $10 \mu \mathrm{M}$ and a residence time of $\sim 1 \mathrm{sec}$. In a kinetochore, redundancy in the organized structure ensures prompt rebinding, but a suitably localized kinase (or other modifying enzyme) can capture and modify the peptide (e.g., the amino-terminal region of Dsn1 or Ndc80) during its transient dissociation, thereby preventing reassociation (Fig. 6). The localized kinase then becomes a disassembly agent.

\section{ACKNOWLEDGMENTS}

We acknowledge support from the Howard Hughes Medical Institute, in which S.C.H. is an Investigator.

\section{REFERENCES}

Akiyoshi B, Nelson CR, Ranish JA, Biggins S. 2009. Analysis of Ipl1-mediated phosphorylation of the Ndc80 kinetochore protein in Saccharomyces cerevisiae. Genetics 183: 1591-1595.

Akiyoshi B, Sarangapani KK, Powers AF, Nelson CR, Reichow SL, Arellano-Santoyo H, Gonen T, Ranish JA, Asbury CL, Biggins S. 2010. Tension directly stabilizes reconstituted kinetochore-microtubule attachments. Nature 468: 576-579.

Akiyoshi B, Nelson CR, Biggins S. 2013. The aurora B kinase promotes inner and outer kinetochore interactions in budding yeast. Genetics 194: 785-789.
Aravamudhan P, Felzer-Kim I, Gurunathan K, Joglekar AP. 2014. Assembling the protein architecture of the budding yeast kinetochore-microtubule attachment using FRET. Curr Biol 24: 1437-1446.

Aravamudhan P, Chen R, Roy B, Sim J, Joglekar AP. 2016. Dual mechanisms regulate the recruitment of spindle assembly checkpoint proteins to the budding yeast kinetochore. $\mathrm{Mol}$ Biol Cell 27: 3405-3417.

Asbury CL, Gestaut DR, Powers AF, Franck AD, Davis TN. 2006. The Dam1 kinetochore complex harnesses microtubule dynamics to produce force and movement. Proc Natl Acad Sci 103: 9873-9878.

Biggins S, Severin FF, Bhalla N, Sassoon I, Hyman AA, Murray AW. 1999. The conserved protein kinase Ipl1 regulates microtubule binding to kinetochores in budding yeast. Genes Dev 13: $532-544$.

Bock LJ, Pagliuca C, Kobayashi N, Grove RA, Oku Y, Shrestha K, Alfieri C, Golfieri C, Oldani A, Dal Maschio M, et al. 2012. Cnn1 inhibits the interactions between the KMN complexes of the yeast kinetochore. Nat Cell Biol 14: 614-624.

Cheeseman IM, Anderson S, Jwa M, Green EM, Kang J, Yates JR III, Chan CS, Drubin DG, Barnes G. 2002. Phospho-regulation of kinetochore-microtubule attachments by the Aurora kinase Ipl1p. Cell 111: 163-172.

Cheeseman IM, Chappie JS, Wilson-Kubalek EM, Desai A. 2006. The conserved KMN network constitutes the core microtubule-binding site of the kinetochore. Cell 127: 983997.

Ciferri C, De Luca J, Monzani S, Ferrari KJ, Ristic D, Wyman C, Stark H, Kilmartin J, Salmon ED, Musacchio A. 2005. Architecture of the human ndc80-hec1 complex, a critical constituent of the outer kinetochore. J Biol Chem 280: 2908829095.

Ciferri C, Pasqualato S, Screpanti E, Varetti G, Santaguida S, Dos Reis G, Maiolica A, Polka J, De Luca JG, De Wulf P, et al. 2008. Implications for kinetochore-microtubule attachment from the structure of an engineered Ndc80 complex. Cell 133: 427-439.

Clarke L, Carbon J. 1980. Isolation of a yeast centromere and construction of functional small circular chromosomes. Nature 287: 504-509.

DeLuca JG, Gall WE, Ciferri C, Cimini D, Musacchio A, Salmon ED. 2006. Kinetochore microtubule dynamics and attachment stability are regulated by Hec1. Cell 127: 969-982.

De Wulf P, McAinsh AD, Sorger PK. 2003. Hierarchical assembly of the budding yeast kinetochore from multiple subcomplexes. Genes Dev 17: 2902-2921.

Dimitrova YN, Jenni S, Valverde R, Khin Y, Harrison SC. 2016. Structure of the MIND complex defines a regulatory focus for yeast kinetochore assembly. Cell 167: 1014-1027 e1012.

Driver JW, Geyer EA, Bailey ME, Rice LM, Asbury CL. 2017. Direct measurement of conformational strain energy in protofilaments curling outward from disassembling microtubule tips. Elife 6: e28433.

Ekeberg M, Lovkvist C, Lan Y, Weigt M, Aurell E. 2013. Improved contact prediction in proteins: Using pseudolikelihoods to infer Potts models. Phys Rev E Stat Nonlin Soft Matter Phys 87: 012707.

Ekeberg M, Hartonen T, Aurell E. 2014. Fast pseudolikelihood maximization for direct-coupling analysis of protein structure from many homologous amino-acid sequences. J Comput Phys 276: 341-356.

Ghaemmaghami S, Huh WK, Bower K, Howson RW, Belle A, Dephoure N, O'Shea EK, Weissman JS. 2003. Global analysis of protein expression in yeast. Nature 425: 737-741.

Grishchuk EL, McIntosh JR. 2006. Microtubule depolymerization can drive poleward chromosome motion in fission yeast. EMBO J 25: 4888-4896.

Hinshaw SM, Harrison SC. 2017. Kinetochore function from the bottom up. Trends Cell Biol. doi: 10.1016/j.tcb.2017.09.002.

Hori T, Amano M, Suzuki A, Backer CB, Welburn JP, Dong Y, McEwen BF, Shang WH, Suzuki E, Okawa K, et al. 2008. CCAN makes multiple contacts with centromeric DNA to 
provide distinct pathways to the outer kinetochore. Cell 135: 1039-1052.

Huis In 't Veld PJ, Jeganathan S, Petrovic A, Singh P, John J, Krenn V, Weissmann F, Bange T, Musacchio A. 2016. Molecular basis of outer kinetochore assembly on CENP-T. Elife 5: e21007.

Joglekar AP, Bouck DC, Molk JN, Bloom KS, Salmon ED. 2006. Molecular architecture of a kinetochore-microtubule attachment site. Nat Cell Biol 8: 581-585.

Kalantzaki M, Kitamura E, Zhang T, Mino A, Novak B, Tanaka TU. 2015. Kinetochore-microtubule error correction is driven by differentially regulated interaction modes. Nat Cell Biol 17: 530.

Kim JO, Zelter A, Umbreit NT, Bollozos A, Riffle M, Johnson R, MacCoss MJ, Asbury CL, Davis TN. 2017. The Ndc80 complex bridges two Dam1 complex rings. Elife 6: e21069.

Koshland DE, Mitchison TJ, Kirschner MW. 1988. Polewards chromosome movement driven by microtubule depolymerization in vitro. Nature 331: 499-504.

Lechner J, Carbon J. 1991. A 240 kd multisubunit protein complex, $\mathrm{CBF} 3$, is a major component of the budding yeast centromere. Cell 64: 717-725.

Li Y, Bachant J, Alcasabas AA, Wang Y, Qin J, Elledge SJ. 2002. The mitotic spindle is required for loading of the DASH complex onto the kinetochore. Genes Dev 16: 183-197.

Maiolica A, Cittaro D, Borsotti D, Sennels L, Ciferri C, Tarricone C, Musacchio A, Rappsilber J. 2007. Structural analysis of multiprotein complexes by cross-linking, mass spectrometry, and database searching. Mol Cell Proteomics 6: 2200 2211.

Malvezzi F, Litos G, Schleiffer A, Heuck A, Mechtler K, Clausen T, Westermann S. 2013. A structural basis for kinetochore recruitment of the Ndc 80 complex via two distinct centromere receptors. EMBO J 32: 409-423.

Maure JF, Komoto S, Oku Y, Mino A, Pasqualato S, Natsume K, Clayton L, Musacchio A, Tanaka TU. 2011. The Ndc80 loop region facilitates formation of kinetochore attachment to the dynamic microtubule plus end. Curr Biol 21: 207-213.

Meluh PB, Yang P, Glowczewski L, Koshland D, Smith MM. 1998. Cse $4 \mathrm{p}$ is a component of the core centromere of Saccharomyces cerevisiae. Cell 94: 607-613.

Miranda JJ, De Wulf P, Sorger PK, Harrison SC. 2005. The yeast DASH complex forms closed rings on microtubules. Nat Struct Mol Biol 12: 138-143.

Miranda JJ, King DS, Harrison SC. 2007. Protein arms in the kinetochore-microtubule interface of the yeast DASH complex. Mol Biol Cell 18: 2503-2510.

Moore MJ, Adams JA, Taylor SS. 2003. Structural basis for peptide binding in protein kinase A. Role of glutamic acid 203 and tyrosine 204 in the peptide-positioning loop. $J$ Biol Chem 278: 10613-10618.

Morcos F, Pagnani A, Lunt B, Bertolino A, Marks DS, Sander C, Zecchina R, Onuchic JN, Hwa T, Weigt M. 2011. Direct-coupling analysis of residue coevolution captures native contacts across many protein families. Proc Natl Acad Sci 108: E1293E1301.

Musacchio A, Desai A. 2017. A molecular view of kinetochore assembly and function. Biology (Basel) 6: E5.

Pekgöz Altunkaya G, Malvezzi F, Demianova Z, Zimniak T, Litos G, Weissmann F, Mechtler K, Herzog F, Westermann S. 2016. CCAN assembly configures composite binding inter- faces to promote cross-linking of $\mathrm{Ndc} 80$ complexes at the kinetochore. Curr Biol 26: 2370-2378.

Petrovic A, Keller J, Liu Y, Overlack K, John J, Dimitrova YN, Jenni S, van Gerwen S, Stege P, Wohlgemuth S, et al. 2016. Structure of the MIS12 complex and molecular basis of its interaction with CENP-C at human kinetochores. Cell 167: 1028-1040.

Powers AF, Franck AD, Gestaut DR, Cooper J, Gracyzk B, Wei RR, Wordeman L, Davis TN, Asbury CL. 2009. The Ndc80 kinetochore complex forms load-bearing attachments to dynamic microtubule tips via biased diffusion. Cell 136: 865 875.

Ramey VH, Wong A, Fang J, Howes S, Barnes G, Nogales E. 2011. Subunit organization in the Dam1 kinetochore complex and its ring around microtubules. Mol Biol Cell 22: 43354342.

Tanaka TU, Rachidi N, Janke C, Pereira G, Galova M, Schiebel E, Stark MJ, Nasmyth K. 2002. Evidence that the Ipl1-Sli15 (Aurora kinase-INCENP) complex promotes chromosome biorientation by altering kinetochore-spindle pole connections. Cell 108: 317-329.

Tanaka TU, Stark MJ, Tanaka K. 2005. Kinetochore capture and bi-orientation on the mitotic spindle. Nat Rev Mol Cell Biol 6: 929-942.

Tanaka K, Kitamura E, Kitamura Y, Tanaka TU. 2007. Molecular mechanisms of microtubule-dependent kinetochore transport toward spindle poles. J Cell Biol 178: 269-281.

Thapa KS, Oldani A, Pagliuca C, De Wulf P, Hazbun TR. 2015. The Mps1 kinase modulates the recruitment and activity of Cnn1(CENP-T) at Saccharomyces cerevisiae kinetochores. Genetics 200: 79-90.

Tytell JD, Sorger PK. 2006. Analysis of kinesin motor function at budding yeast kinetochores. J Cell Biol 172: 861-874.

Valverde R, Ingram J, Harrison SC. 2016. Conserved tetramer junction in the kinetochore Ndc80 complex. Cell Rep 17: 1915-1922.

van Hooff JJE, Snel B, Kops G. 2017. Unique phylogenetic distributions of the Ska and Dam1 complexes support functional analogy and suggest multiple parallel displacements of Ska by Dam1. Genome Biol Evol 9: 1295-1303.

Wang HW, Long S, Ciferri C, Westermann S, Drubin D, Barnes G, Nogales E. 2008. Architecture and flexibility of the yeast Ndc80 kinetochore complex. J Mol Biol 383: 894-903.

Wei RR, Sorger PK, Harrison SC. 2005. Molecular organization of the Ndc80 complex, an essential kinetochore component. Proc Natl Acad Sci 102: 5363-5367.

Wei RR, Schnell JR, Larsen NA, Sorger PK, Chou JJ, Harrison SC. 2006. Structure of a central component of the yeast kinetochore: The Spc24p/Spc25p globular domain. Structure 14: 1003-1009.

Wei RR, Al-Bassam J, Harrison SC. 2007. The Ndc80/HEC1 complex is a contact point for kinetochore-microtubule attachment. Nat Struct Mol Biol 14: 54-59.

Westermann S, Avila-Sakar A, Wang HW, Niederstrasser H, Wong J, Drubin DG, Nogales E, Barnes G. 2005. Formation of a dynamic kinetochore-microtubule interface through assembly of the Dam1 ring complex. Mol Cell 17: 277-290.

Winey M, Mamay CL, O’Toole ET, Mastronarde DN, Giddings TH Jr, McDonald KL, McIntosh JR. 1995. Three-dimensional ultrastructural analysis of the Saccharomyces cerevisiae mitotic spindle. J Cell Biol 129: 1601-1615. 


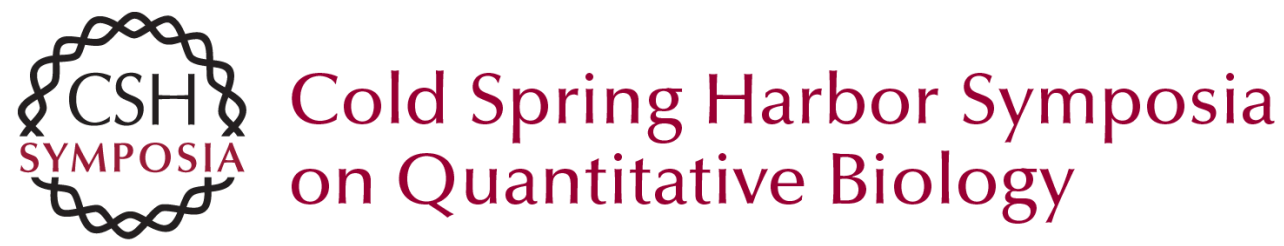

\section{Molecular Structures of Yeast Kinetochore Subcomplexes and Their Roles in Chromosome Segregation}

Simon Jenni, Yoana N. Dimitrova, Roberto Valverde, et al.

Cold Spring Harb Symp Quant Biol 2017 82: 83-89 originally published online November 22, 2017

Access the most recent version at doi:10.1101/sqb.2017.82.033738

References This article cites 55 articles, 20 of which can be accessed free at: http://symposium.cshlp.org/content/82/83.full.html\#ref-list-1

Creative This article is distributed under the terms of the

Commons http://creativecommons.org/licenses/by-nc/4.0/, which permits reuse and

License redistribution, except for commercial purposes, provided that the original author and source are credited.

Email Alerting Receive free email alerts when new articles cite this article - sign up in Service the box at the top right corner of the article or click here. 